\title{
Mineralocorticoid receptor: a critical player in vascular remodeling
}

\author{
DUAN ShengZhong \\ Key Laboratory of Nutrition and Metabolism, Institute for Nutritional Sciences, Shanghai Institutes for Biological Sciences, Chinese Academy \\ of Sciences, Shanghai 200031, China
}

Received April 29, 2014; accepted June 6, 2014

\begin{abstract}
Vascular remodeling is a pathological condition with structural changes of blood vessels. Both inside-out and outside-in hypothesis have been put forward to describe mechanisms of vascular remodeling. An integrated model of these two hypotheses emphasizes the importance of immune cells such as monocytes/macrophages, T cells, and dendritic cells. These immune cells are at the center stage to orchestrate cellular proliferation, migration, and interactions of themselves and other vascular cells including endothelial cells (ECs), vascular smooth muscle cells (VSMCs), and fibroblasts. These changes on vascular wall lead to inflammation and oxidative stress that are largely responsible for vascular remodeling. Mineralocorticoid receptor (MR) is a classic nuclear receptor. MR agonist promotes inflammation and oxidative stress and therefore exacerbates vascular remodeling. Conversely, MR antagonists have the opposite effects. MR has direct roles on vascular cells through non-genomic or genomic actions to modulate inflammation and oxidative stress. Recent studies using genetic mouse models have revealed that MR in myeloid cells, VSMCs and ECs all contribute to vascular remodeling. In conclusion, data in the past years have demonstrated that MR is a critical control point in modulating vascular remodeling. Studies will continue to provide evidence with more detailed mechanisms to support this notion.
\end{abstract}

vascular remodeling, mineralocorticoid receptor, immune cell, endothelial cell, vascular smooth muscle cell, fibroblast

Citation: Duan SZ. Mineralocorticoid receptor: a critical player in vascular remodeling. Sci China Life Sci, 2014, 57: 809-817, doi: $10.1007 / \mathrm{s} 11427-014-4691-5$

What is vascular remodeling? A simple and straightforward and possibly the most correct answer is that any structural changes of blood vessels are called vascular remodeling [1]. The causes of these changes range from pressure, flow and atherosclerotic stimuli, to mechanical injury such as balloon angioplasty and stent implantation during endovascular interventions. The changes can be on the vascular wall, the luminal diameter, or both. Although the changes on the vascular wall and luminal diameter often happen together, there are situations that only one of them alters. For example, Glagov and colleagues [2] found that the luminal diameter of atherosclerotic human coronaries remained constant until the percent stenosis exceeded $40 \%$, whereas the vas- cular wall had dramatically changed because of atherosclerosis. These structural changes are often accompanied by functional alterations of the blood vessels. Therefore, vascular remodeling is closely associated with the pathophysiology of a series of cardiovascular diseases such as atherosclerosis and restenosis, cerebrovascular diseases such as stroke, as well as hypertension. In this review, I will discuss the roles of mineralocorticoid receptor, a member of the nuclear receptor superfamily that controls numerous critical aspects of cellular functions under physiological or pathophysiological conditions, in vascular remodeling. This article will not cover vascular remodeling in embryonic development or tumor growth, even though structural changes of blood vessels also happen in both processes $[3,4]$.

email: duansz2008@gmail.com 


\section{The mechanisms of vascular remodeling: the inside-out and outside-in hypotheses}

At least two different models have been put forward to describe the process of vascular remodeling, the inside-out hypothesis and outside-in hypothesis [5,6]. Traditionally, the vascular remodeling is considered as an inside-out process. In this model, loss of endothelial integrity is a starting point followed by expression of surface adhesion molecules and inflammatory mediators that propel adhesion and transmigration of circulating monocytes into vascular wall. The causes of endothelial injury include mechanical injury, chemical stimuli, insulin resistance and associated glucose intolerance, dyslipidemia, hypertension, increased oxidation, thrombosis, and vascular inflammation [7]. Monocytes further differentiate into macrophages in the vascular wall and traditionally this process had been considered as a continuous one for the presence of monocytes/macrophages to function without excessive proliferation in the progression of vascular remodeling [8]. However, recent studies have demonstrated that in the process of atherosclerosis macrophages in the plaque maintain its population largely by proliferation after the initial migration of monocytes [9]. Either way, macrophages in the vascular wall interact with vascular smooth muscle cells (VSMCs) in the media directly or through secreted inflammatory chemokines, cytokines and reactive oxygen species (ROS). Consequently, VSMCs proliferate and migrate to subendothelial space and participate in repairing of damaged endothelium, formation of plaque or neointima, and vascular remodeling. In addition, VSMCs may dedifferentiate into myofibroblasts, contributing to vascular remodeling [5]. Other immune cells such as neutrophils, dendritic cells (DCs), T cells, B cells, and mast cells also play important roles in development and progression of atherosclerosis [10]. Fibroblasts are in general considered as bystanders in this model.

In contrast, the outside-in hypothesis proposes that injury and immune response are initiated in the adventitia and progressed inward to the media and intima $[5,6]$. In this model, fibroblasts in the adventitia are highly active. In response to vascular stress or injury, adventitia fibroblasts are often among the first cells to be activated in the vascular wall, accompanied by activation of immune cells such as $\mathrm{T}$ cells, DCs, and macrophages. Besides their traditional function of secreting extracellular matrix (ECM), activated and highly proliferated fibroblasts play central roles in vascular remodeling through the following aspects. First, these activated fibroblasts are considered as capable of executing immune functions. They express receptors such as toll-like receptors (TLRs) and NOD-like receptors (NLRs) to mediate immune response. Further, they produce chemokines and cytokines such as monocyte chemotactic protein-1 (MCP1), regulated upon activation, normal T cell expressed and secreted (RANTES), interleukin 6 (IL6), and stromal cell-derived factor 1 (SDF-1). Secondly, fibroblasts interact with activated immune cells (T cells, DCs, and macrophages), ECs and VSMCs, either directly through cell-cell interactions or through chemokines, cytokines and ROS. Macrophages in this model are mostly regarded as resident ones or being differentiated from monocytes recruited from the vasa vasorum of the adventitia locally. In addition, proliferated fibroblasts can migrate to the media and intima, or differentiate into myofibroblasts that subsequently migrate to the media and intima, contributing to vascular remodeling [11].

\section{An integrated model of mechanisms: immune cells at the center stage of vascular remodeling}

The two seemly contrasting hypotheses can come together and therefore I propose an integrated model for the mechanisms of vascular remodeling (Figure 1). For instance, in the procedure of stent, dramatic endothelial damages are accompanied by stretch stimulus of the whole blood vessels including the adventitia. It is possible that both mechanisms execute and propel each other. The inside-out process leads to macrophage accumulation in the subendothelial space and interacting with endothelial cells (ECs) and VSMCs. The macrophages may also accumulate in the adventitia and interact with fibroblasts and other immune cells, which in turn induce an outside-in process. The adventitia may also sense the stretch at the same time of endothelial injury and ignite an outside-in process by activating local macrophages, fibroblasts, T cells, and DCs. Of course, it is possible that the inside-out process is more dominant in pathological conditions such as atherosclerosis and the outside-in process is more dominant in others such as hypertension.

In either inside-out, outside-in, or integrated hypothesis, the immune cells play central roles in vascular remodeling. They respond to stress and injury, migrate into or proliferate in the vascular wall, and interact with other vascular cells. Monocytes/macrophages are a subset of these immune cells that have drawn much attention. Interestingly, macrophages predominantly accumulate in the adventitia in various models of vascular remodeling such as angiotensin II (Ang II)induced, pressure overload-induced, and wire injuryinduced [12-14], although macrophages-derived foam cells largely accumulate in the subendothelial area to form plaque in atherosclerosis. This implies that even if the macrophages come from the systemic circulation according to the inside-out hypothesis, these cells can ultimately dwell in the adventitia and interact with cells including fibroblasts and other immune cells there. These immune cells (monocytes/macrophages, T cells, DCs) interact with each other and with ECs, VSMCs, and fibroblasts, driving the whole process of vascular remodeling (Figure 1). 


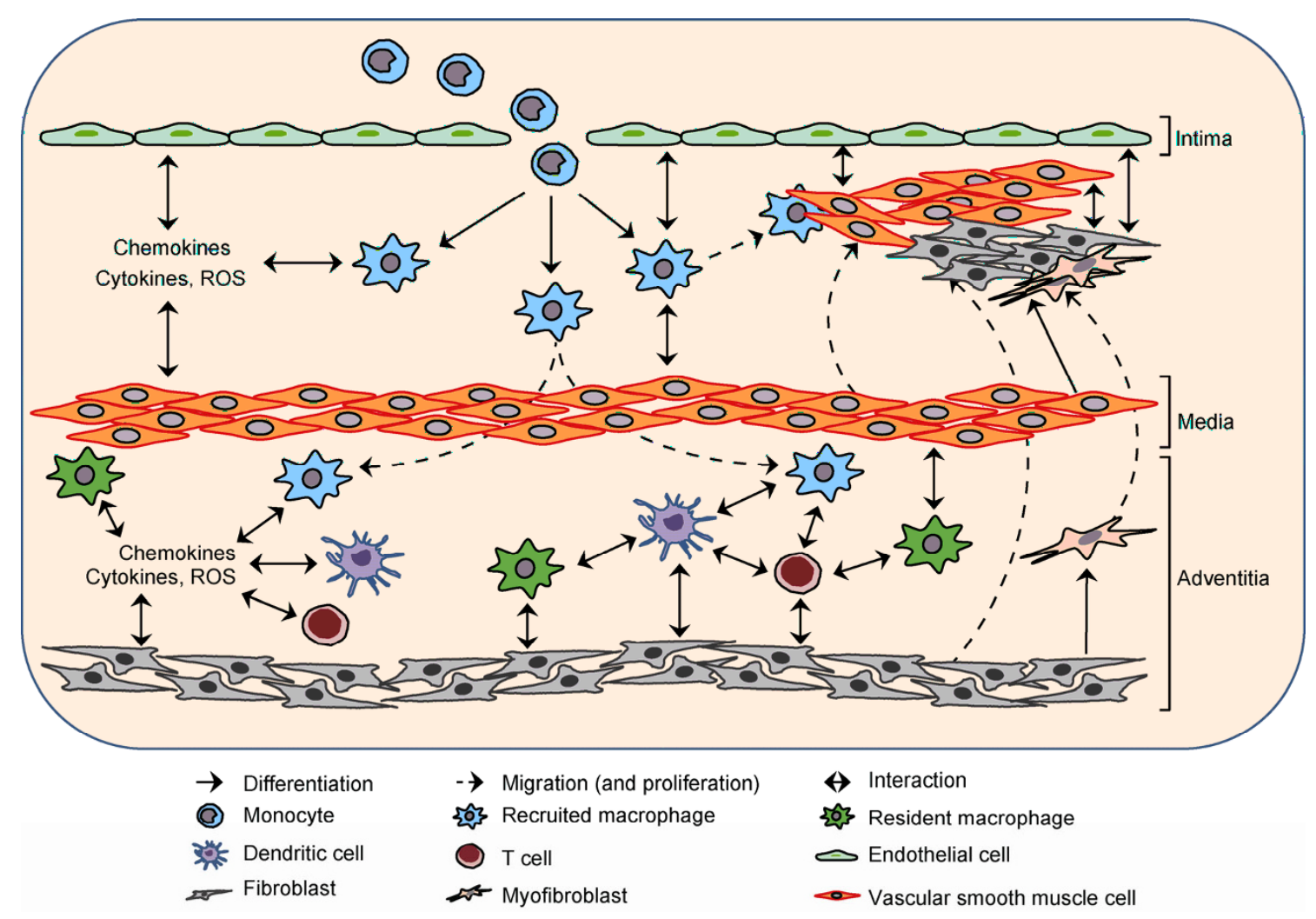

Figure 1 (color online) An integrated model for mechanisms of vascular remodeling. This proposed model is an integration of the inside-out hypothesis and outside-in hypothesis. Endothelial injuries initiate an inside-out process that leads to macrophage accumulation in the subendothelial space and interacting with endothelial cells and vascular smooth muscle cells through cell-cell interactions or secreted chemokines, cytokines and reactive oxygen species (ROS). Macrophages also migrate to and accumulate in the adventitia and interact with fibroblasts and other immune cells, which in turn induce an outside-in process. Simultaneously, the adventitia senses the stress and ignites an outside-in process by activating local macrophages, fibroblasts, $\mathrm{T}$ cells, and dendritic cells, all of which ultimately propel the inside-out process. In such way, the inside-out process and outside-in process act in concert and reinforce each other, being orchestrated by immune cells at the center stage and driving the whole course of vascular remodeling.

\section{Mineralocorticoid receptor and vascular re- modeling}

Mineralocorticoid receptor (MR) is a classic steroid receptor in the nuclear receptor superfamily [15]. To exert its genomic actions, MR binds to mineralocorticoids (primarily aldosterone), translocates into nucleus, binds as homodimers to response elements of target genes, and therefore regulates their expression. The target genes of MR include those important in water-electrolyte homeostasis and blood pressure regulation, inflammation, oxidative stress, and fibrosis [16]. Recent studies have revealed that MR also has non-genomic actions by interactions with signaling pathways such as epidermal growth factor (EGF)/EGFR pathway and Ang II/type 1 Ang II receptors (AT1R) pathway [16].

It is now well accepted that the function of MR is far beyond its traditional roles in epithelial cells to control water-electrolyte homeostasis and blood pressure. Clinical studies have continuously and convincingly demonstrated the effectiveness of MR antagonists (spironolactone and eplerenone) in treating heart failure patients [17-19].
Mounting evidence from basic research has illustrated the critical functions of this nuclear receptor in cardiovascular system.

MR is expressed in vascular cells and the vasculature is generally regarded as an aldosterone-responsive tissue [20]. The concentration of glucocorticoids in the bloodstream is over 100 times higher than that of aldosterone and MR binds to both with similar affinity. Therefore, MR has the potential to bind to aldosterone only in tissues that express $11 \beta$-hydroxysteroid dehydrogenase type 2 (11ßHSD2), an enzyme that inactivates glucocorticoids. Traditionally, kidney and colon are considered as aldosterone sensitive tissues because of the high level of expression of $11 \beta \mathrm{HSD} 2$. More recent studies, however, have revealed that vascular cells including VSMCs and ECs express $11 \beta \mathrm{HSD} 2$ as well, indicating that blood vessels are aldosterone-responsive. It remains to be determined whether this enzyme is expressed in macrophages or other immune cells.

\subsection{Aldosterone and vascular remodeling}

Both epidemiological studies and animal studies have shown the importance of aldosterone in vascular remodeling. 
Circulating level of aldosterone is an independent predictor of cardiovascular ischemia according to epidemiological studies [21-23]. When blood pressure is comparable, patients with hyperaldosteronism have drastically increased risk of stroke and myocardial infarction. These results suggest that aldosterone may promote atherosclerosis and plaque rupture in humans independent of its impact on blood pressure and that aldosterone and MR play important roles in vascular biology. Animal studies have also shown that aldosterone increases atherosclerosis in apoE knockout mice and this effect can be attenuated by blood pressure reduction [24]. More recently, aldosterone was found to promote early atherosclerosis and plaque inflammation in apoE knockout mice at a lower dose that did not elevate blood pressure, indicating that aldosterone may also directly affect atherosclerosis in animal models independent of its impact on blood pressure [25]. In addition, aldosterone infusion induces vascular fibrosis and remodeling in rats in the setting of high salt [26]. In a swine model of balloon angioplasty aldosterone tends to exacerbate collagen accumulation and constrictive remodeling of coronary arteries [27].

Aldosterone increases inflammation and oxidative stress in different vascular cells [26,28,29]. MR activation stimulates infiltration of inflammatory cells including monocytes, macrophages and lymphocytes and promotes expression of inflammatory markers in the vasculature. Meanwhile, MR activation upregulates oxidative stress by increasing expression and activity of NADPH oxidases (NOX), production of ROS, and mitochondrial electron transport uncoupling. Given the vicious cycle of inflammation and oxidative stress, excessive aldosterone stimulates the two components that propel each other, promoting proliferation and migration of vascular cells, ECM deposition, and ultimately vascular remodeling.

\subsection{MR antagonists and vascular remodeling}

Both eplerenone and spironolactone attenuate collagen accumulation and constrictive remodeling of coronary arteries without affecting the size of neointima in a swine model of balloon angioplasty [27]. In a swine model of stenting, eplerenone suppresses neointima formation, vascular fibrosis and remodeling [30]. Spironolactone inhibits Ang IIinduced increase in media-to-lumen ratio of mesenteric resistance arteries and decrease in acetylcholine-induced vasodilation while decreasing blood pressure and oxidative stress in a rat model [31]. Similarly, structural and functional abnormalities induced by high salt in spontaneously hypertensive rats were inhibited by eplerenone [32]. Subsequent data using Ang II overproducing transgenic mice showed that spironolactone improved vascular remodeling independent of blood pressure [33]. More recently, spironolactone has been shown to attenuate pulmonary vascular resistance and pulmonary vascular remodeling in an experimental pulmonary hypertension mouse model [34]. In addition, MR antagonists can inhibit atherosclerosis in different animal models [35-37]. Mechanistically, blockade of MR by antagonists inhibits oxidative stress and/or inflammation and therefore breaks the vicious cycle. These data further demonstrated the importance of MR in vascular remodeling, providing foundations for further investigations on cell type-specific functions of MR.

\subsection{Myeloid MR and vascular remodeling}

Myeloid cells play important roles in atherosclerosis and restenosis. Functions of myeloid MR in vascular remodeling have been studied using a knockout mouse model. When challenged with a combination of a NOS inhibitor, $N^{G}$-nitroL-arginine methyl ester (L-NAME), and Ang-II, myeloid MR knockout (MMRKO) mice had decreased vascular fibrosis and remodeling, in addition to suppressed cardiac remodeling [12]. Macrophage infiltration in heart and aorta was almost completely blocked by myeloid MR deletion. Intriguingly, blood pressure was higher in the MMRKO mice, suggesting that the cardiovascular protection of myeloid MR deficiency was not because of its impact on blood pressure. Further, MR activation by aldosterone stimulated pro-inflammatory M1 polarization of macrophages and that MRKO or MR antagonists resulted in anti-inflammatory M2 polarization of macrophages by regulating a host of M1 and M2 genes. These M1 genes include interleukin $1 \beta$ (IL1 $\beta$ ), IL6, IL12, monocyte chemotactic protein-1 (MCP1), regulated upon activation, normal $\mathrm{T}$ cell expressed and secreted (RANTES), and tumor necrosis factor- $\alpha$ (TNF $\alpha$ ). M2 genes include arginase 1 (Arg1), chemokine (C-C motif) ligand 7 (CCL7), CCL17, found in inflammatory zone 1 (FIZZ1), coagulation factor XIII a1 (F13a1), IL10, macrophage scavenger receptor (MSR), and T-lymphocytederived eosinophil chemotactic factor (YM1). Additionally, MRKO or MR antagonists decreased expression of profibrotic genes including plasminogen activator inhibitor-1 (PAI1), transforming growth factor $\beta$ (TGF $\beta$ ), as well as increased expression of anti-fibrotic genes including cysteine-rich, angiogenic inducer 61 (CYR61), high-temperature requirement A1 (HTRA1), and pyruvate dehydrogenase kinase 4 (PDK4). More recently, these phenotypes were also demonstrated in human monocytes/macrophages [38,39]. Therefore, MR acts as a strong control point for macrophage polarization that may ultimately explain the beneficial effects of MRKO on vascular remodeling (Figure 2 ). The detailed mechanisms are still under intensive investigations.

\subsection{VSMC MR and vascular remodeling}

VSMC-specific knockout of MR (SMRKO) effectively 


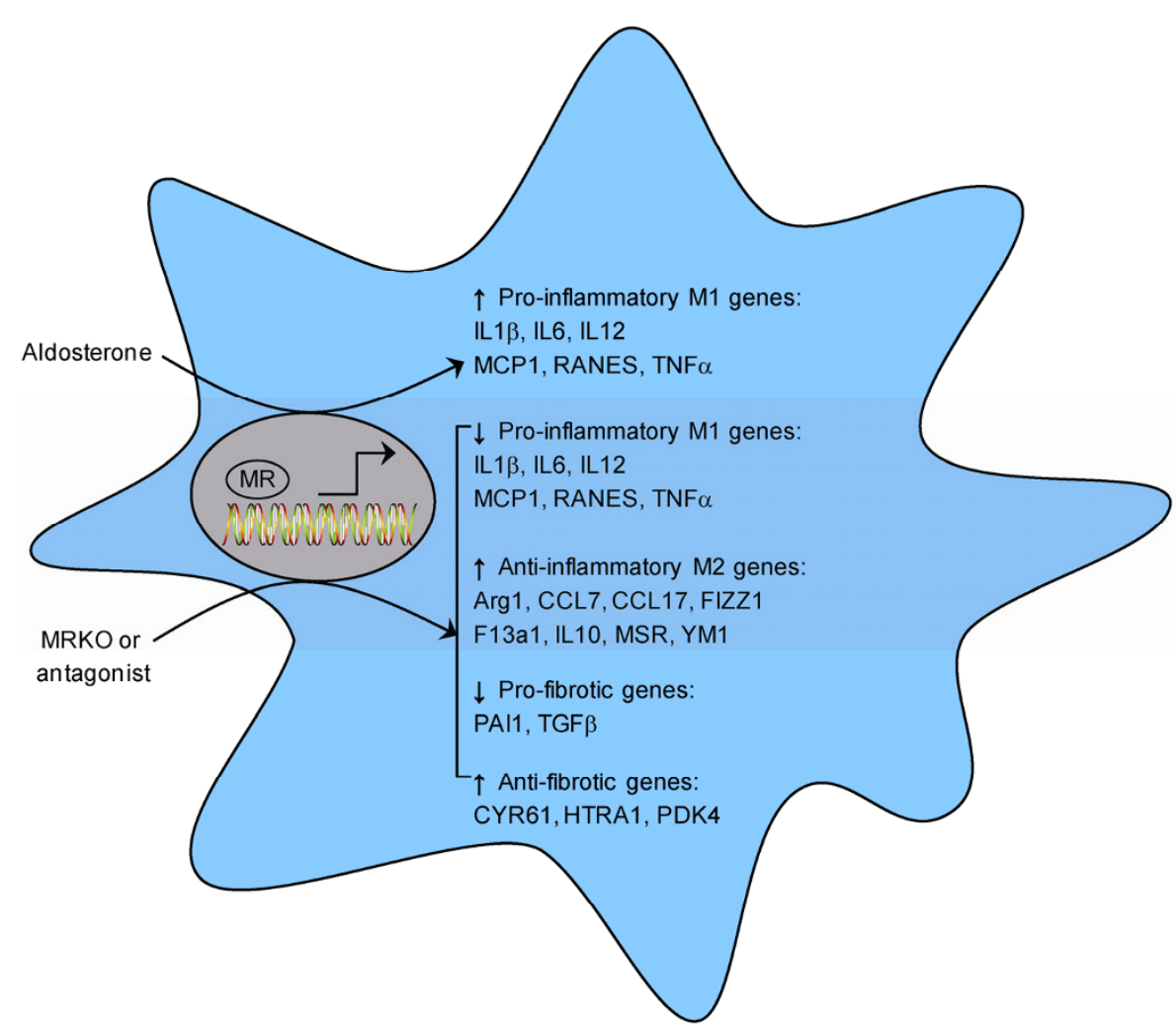

Figure 2 (color online) Functions of macrophage mineralocorticoid receptor (MR) in vascular remodeling. Aldosterone promotes expression of pro-inflammatory genes and drives a classically activated (M1) phenotype of macrophages. Conversely, MR deficiency (MRKO) or antagonist suppresses pro-inflammatory gene expression while promotes anti-inflammatory gene expression and therefore drives an alternatively activated (M2) phenotype. MRKO or antagonist also decreases pro-fibrotic gene expression while increases anti-fibrotic gene expression. These effects ultimately translate into deleterious impacts of aldosterone and beneficial impacts of MRKO or antagonist on vascular remodeling. IL1 $\beta$ : interleukin 1 $\beta$; MCP1: monocyte chemotactic protein-1; RANTES: regulated upon activation, normal T cell expressed and secreted; TNF $\alpha$ : tumor necrosis factor- $\alpha$; Arg1: arginase 1; CCL7: chemokine (C-C motif) ligand 7; FIZZ1: found in inflammatory zone 1; F13a1: coagulation factor XIII a1; MSR: macrophage scavenger receptor; YM1: T-lymphocyte-derived eosinophil chemotactic factor; PAI1: plasminogen activator inhibitor-1; TGF $\beta$ : transforming growth factor $\beta$; CYR61: cysteine-rich, angiogenic inducer, 61; HTRA1: high-temperature requirement A1; PDK4: pyruvate dehydrogenase kinase 4.

blocked age-associated hypertension through decreasing vascular myogenic tone and vasoconstriction response, without alterations in vascular structure or renal function [40]. However, in a wire-induced carotid injury model in combination with aldosterone infusion, SMRKO dramatically decreased VSMC proliferation and vascular fibrosis that were induced by aldosterone or injury alone, through a placental growth factor/type 1 vascular endothelial growth factor receptor (VEGFR1) pathway [41].

At the cellular level, MR plays direct roles in VSMCs through rapid non-genomic pathways or slower genomic effects (Figure 3). A large amount of data have been generated on studying the non-genomic actions of MR in VSMCs $[20,26,42,43]$. Aldosterone has been shown to rapidly transactivate EGFR or platelet-derived growth factor receptor (PDGFR), which are followed by activation of nonreceptor tyrosine kinase c-Src. Subsequently, c-Src phosphorylates and activates mitogen-activated protein kinases (MAPK) including ERK, JNK, and P38. Meanwhile, c-Src upregulates redox pathways through activation of NOX. Genomic actions of MR have also been reported in VSMCs.
MR has been demonstrated to regulate expression of genes such as dual specificity protein phosphatase 1 (Dusp1), plasminogen activator inhibitor-1 (PAI-1), connective tissue growth factor (CTGF), collagen I, and collagen III [20]. Importantly, these phenotypes of aldosterone can be inhibited by MR antagonists, indicating the dependency of MR. Furthermore, both non-genomic and genomic actions are potentiated by crosstalk between Ang II/AT1R pathway and aldosterone/MR pathway [20,42]. In the end, these non-genomic and genomic actions of MR in VSMCs lead to inflammation, oxidative stress, proliferation, migration, and ECM deposition, contributing to vascular remodeling.

\subsection{EC MR and vascular remodeling}

Either at baseline or stimulated with Ang II or endothelin 1, endothelial cell-specific MR overexpression (ECMROE) mice had significantly elevated blood pressure that could be reversed by MR antagonist. Furthermore, the resistance arteries from ECMROE mice had increased contractile response to vasoconstrictors. Intriguingly, despite these func- 


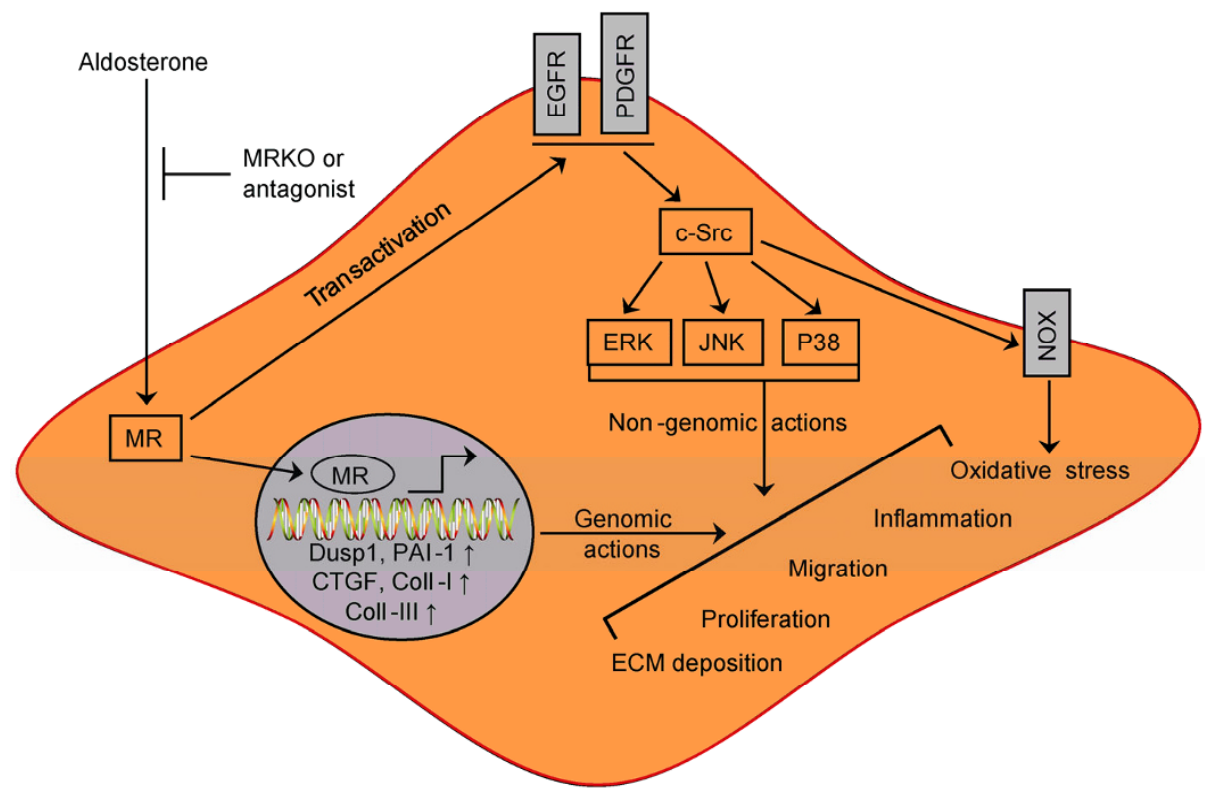

Figure 3 (color online) Functions of vascular smooth muscle cell mineralocorticoid receptor (MR) in vascular remodeling. On one hand, aldosterone binds to MR and regulates expression of genes such as dual specificity protein phosphatase 1 (Dusp1), plasminogen activator inhibitor-1 (PAI-1), connective tissue growth factor (CTGF), collagen I (Coll-I), and collagen III (Coll-III), leading to genomic effects in vascular smooth muscle cells (VSMCs). On the other hand, MR transactivates epidermal growth factor receptor (EGFR) or platelet-derived growth factor receptor (PDGFR) and subsequently activates a non-genomic cascade involving non-receptor tyrosine kinase c-Src and mitogen-activated protein kinases (MAPK) including ERK, JNK, and P38. c-Src also activates NADPH oxidases (NOX) directly. These non-genomic and genomic actions of MR in VSMCs lead to inflammation, oxidative stress, proliferation, migration, and extracellular matrix (ECM) deposition, contributing to vascular remodeling. MR deficiency (MRKO) in VSMCs or antagonist of MR largely blocks the effects of aldosterone.

tional changes, no morphological alterations were observed between ECMROE mice and littermate control mice [44]. EC MR knockout (ECMRKO) prevented endothelial dysfunction, measured by endothelium-dependent vasodilation of aortic rings, in mouse models of both obesity and aldosterone infusion [45]. Further experiments showed that ECMRKO could prevent aldosterone-induced endothelial dysfunction in aortas but not in mesenteric arteries, suggesting differential function of endothelial MR in large conduit arteries vs. resistance vessels [46]. Blood pressure was not different between ECMRKO mice at baseline or stimulated with DOCA. The functions of endothelial MR in vascular remodeling are worth further exploration in different models and with more mechanistic depth.

At the cellular level, MR also plays direct roles in ECs (Figure 4). In vascular ECs, aldosterone has been shown to promote expression of intercellular adhesion molecule-1 (ICAM-1) and vascular cell adhesion molecule-1 (VCAM-1) and therefore adhesion of leukocytes [47,48]. Aldosterone induces ROS generation in ECs by activation of NOX through a c-Src/Rac1 pathway or by suppression of glucose-6-phosphate dehydrogenase (G6PD) activity, in an MR-dependent manner [49,50]. Further, although there are conflicting data [51], MR activation has been shown to decrease endothelial nitric oxide synthase (eNOS) and production of nitric oxide (NO) by activation of protein phosphatase 2A (PP2A) [20,52]. In addition, aldosterone has been shown to activate ERK through phosphoinositide 3-kinase
(PI3K) in ECs [51]. A link between aldosterone and EGFR has also been proposed in ECs [43]. It is likely that in these cells aldosterone also transactivates EGFR that subsequently activates c-Src, a pathway that is operated in VSMCs. It remains to be determined whether PP2A and PI3K are activated by $\mathrm{c}-\mathrm{Src}$, which has been reported in other systems, or by other mechanisms. Consequently, MR activation in ECs promotes inflammation, oxidative stress, and endothelial dysfunction, contributing to vascular remodeling.

\section{Conclusion and future directions}

Vascular remodeling is a complicated pathological process involving multiple types of cells. Inflammation and oxidative stress are the major driving forces of vascular remodeling. Studies in the past years have demonstrated that MR is a critical control point to break the vicious cycle of inflammation and oxidative stress in various vascular cells. These data support that MR is a potential target for modulating vascular remolding and warrant clinical studies to test therapeutic effects of MR antagonists that have already been used for heart failure patients.

MR will continue to be an interesting and important research topic in vascular remodeling with many questions unanswered. For instance, functions of MR in $\mathrm{T}$ cells and DCs in vascular remodeling are still to be investigated. MR is abundantly expressed in DCs and may be also expressed 


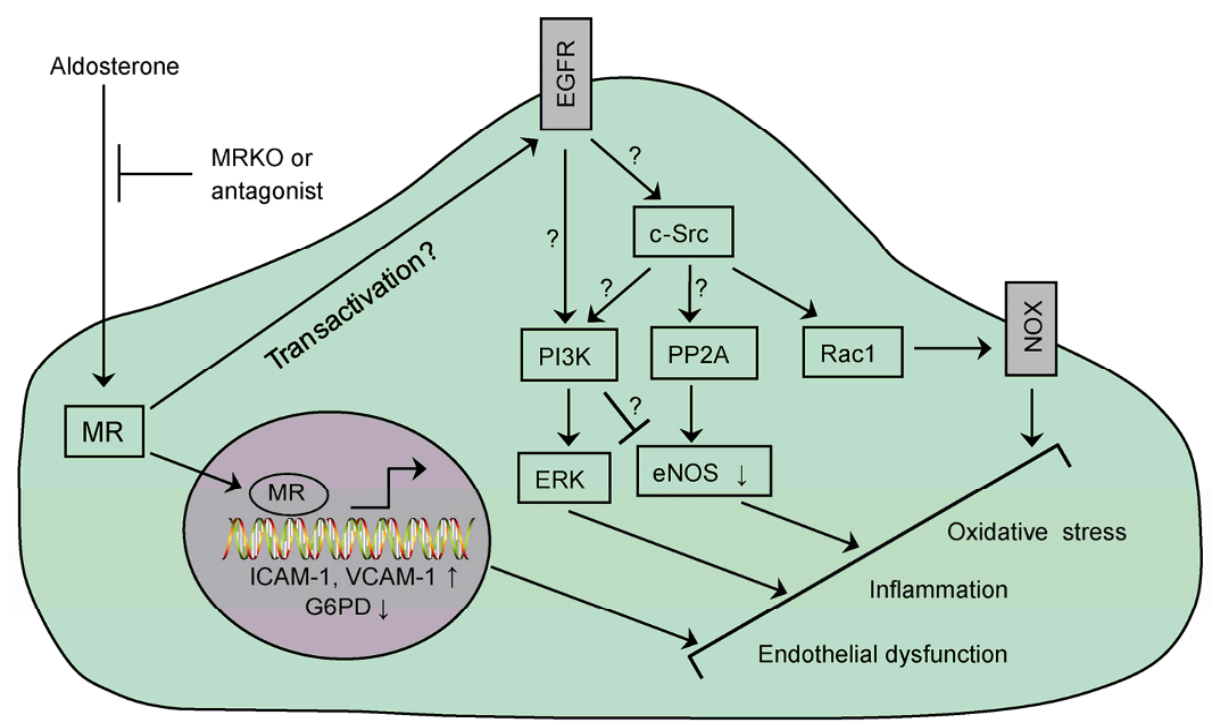

Figure 4 (color online) Functions of endothelial mineralocorticoid receptor (MR) in vascular remodeling. Aldosterone binds to MR and regulates expression of genes such as intercellular adhesion molecule-1 (ICAM-1), vascular cell adhesion molecule-1 (VCAM-1), and glucose-6-phosphate dehydrogenase (G6PD) in endothelial cells (ECs). The non-genomic effects of MR in ECs are less clear. MR may transactivate epidermal growth factor receptor (EGFR) and subsequently activate a non-genomic cascade involving non-receptor tyrosine kinase c-Src and/or phosphoinositide 3-kinase (PI3K)/ERK. c-Src activates NADPH oxidases (NOX) through Rac1 and may also activate protein phosphatase $2 \mathrm{~A}$ (PP2A) and/or PI3K. PP2A can decrease endothelial nitric oxide synthase (eNOS) and production of nitric oxide. These effects of MR in ECs lead to inflammation, oxidative stress, and endothelial dysfunction, contributing to vascular remodeling. MR deficiency (MRKO) in ECs or antagonist of MR largely blocks the effects of aldosterone. The question marks (?) indicate possible links that remain to be further confirmed.

in $\mathrm{T}$ cells at a much lower level [53,54]. It has been shown that aldosterone promotes activation of $\mathrm{CD}^{+} \mathrm{T}$ cells and Th17 polarization of $\mathrm{CD}^{+} \mathrm{T}$ cells and that these effects are dependent on activation of MR and modulation of DC function by aldosterone [54]. More recently, it has been reported that MR activation reduces the amount of circulating naïve $\mathrm{CD}^{+}$and $\mathrm{CD}^{+} \mathrm{T}$ cells in human subjects, further suggesting important functions of MR in $\mathrm{T}$ cells [55]. However, direct roles of MR in T cells or DCs have not been reported in any setting of vascular remodeling. In addition, detailed molecular mechanisms of MR in immune cells and vascular cells need to be further delineated.

This work was supported by grants from the One Hundred Talents Program of the Chinese Academy of Sciences (2012OHTP06), the National Basic Research Program of China (2012CB524900), and the National Natural Science Foundation of China (91339110, 31371153, 31171133).

1 Gibbons GH, Dzau VJ. The emerging concept of vascular remodeling. N Engl J Med, 1994, 330: 1431-1438

2 Glagov S, Weisenberg E, Zarins CK, Stankunavicius R, Kolettis GJ. Compensatory enlargement of human atherosclerotic coronary arteries. N Engl J Med, 1987, 316: 1371-1375

3 Jones EA, le Noble F, Eichmann A. What determines blood vessel structure? Genetic prespecification vs. hemodynamics. Physiology, 2006, 21: 388-395

4 Potente M, Gerhardt H, Carmeliet P. Basic and therapeutic aspects of angiogenesis. Cell, 2011, 146: 873-887

5 Stenmark KR, Yeager ME, El Kasmi KC, Nozik-Grayck E, Gerasimovskaya EV, Li M, Riddle SR, Frid MG. The adventitia: essential regulator of vascular wall structure and function. Annu Rev
Physiol, 2013, 75: 23-47

6 Stenmark KR, Frid MG, Yeager M, Li M, Riddle S, McKinsey T, El Kasmi KC. Targeting the adventitial microenvironment in pulmonary hypertension: a potential approach to therapy that considers epigenetic change. Pulmon circulat, 2012, 2: 3-14

7 Duan SZ, Usher MG, Mortensen RM. Peroxisome proliferatoractivated receptor-gamma-mediated effects in the vasculature. Circ Res, 2008, 102: 283-294

8 Swirski FK, Pittet MJ, Kircher MF, Aikawa E, Jaffer FA, Libby P, Weissleder R. Monocyte accumulation in mouse atherogenesis is progressive and proportional to extent of disease. Proc Natl Acad Sci USA, 2006, 103: 10340-10345

9 Robbins CS, Hilgendorf I, Weber GF, Theurl I, Iwamoto Y, Figueiredo JL, Gorbatov R, Sukhova GK, Gerhardt LM, Smyth D, Zavitz CC, Shikatani EA, Parsons M, van Rooijen N, Lin HY, Husain M, Libby P, Nahrendorf M, Weissleder R, Swirski FK. Local proliferation dominates lesional macrophage accumulation in atherosclerosis. Nat Med, 2013, 19: 1166-1172

10 Weber C, Noels H. Atherosclerosis: current pathogenesis and therapeutic options. Nat Med, 2011, 17: 1410-1422

11 Sartore S, Chiavegato A, Faggin E, Franch R, Puato M, Ausoni S, Pauletto P. Contribution of adventitial fibroblasts to neointima formation and vascular remodeling: from innocent bystander to active participant. Circ Res, 2001, 89: 1111-1121

12 Usher MG, Duan SZ, Ivaschenko CY, Frieler RA, Berger S, Schutz G, Lumeng CN, Mortensen RM. Myeloid mineralocorticoid receptor controls macrophage polarization and cardiovascular hypertrophy and remodeling in mice. J Clin Invest, 2010, 120: 3350-3364

13 Sata M, Maejima Y, Adachi F, Fukino K, Saiura A, Sugiura S, Aoyagi T, Imai Y, Kurihara H, Kimura K, Omata M, Makuuchi M, Hirata Y, Nagai R. A mouse model of vascular injury that induces rapid onset of medial cell apoptosis followed by reproducible neointimal hyperplasia. J Mol Cell Cardiol, 2000, 32: 2097-2104

14 Nagata K, Obata K, Xu J, Ichihara S, Noda A, Kimata H, Kato T, Izawa $\mathrm{H}$, Murohara $\mathrm{T}$, Yokota $\mathrm{M}$. Mineralocorticoid receptor antagonism attenuates cardiac hypertrophy and failure in low- 
aldosterone hypertensive rats. Hypertension, 2006, 47: 656-664

15 Evans RM, Mangelsdorf DJ. Nuclear Receptors, RXR, and the Big Bang. Cell, 2014, 157: 255-266

16 Lastra G, Dhuper S, Johnson MS, Sowers JR. Salt, aldosterone, and insulin resistance: impact on the cardiovascular system. Nat Rev Cardiol, 2010, 7: 577-584

17 Pitt B, Remme W, Zannad F, Neaton J, Martinez F, Roniker B, Bittman R, Hurley S, Kleiman J, Gatlin M. Eplerenone, a selective aldosterone blocker, in patients with left ventricular dysfunction after myocardial infarction. N Engl J Med, 2003, 348: 1309-1321

18 Pitt B, Zannad F, Remme WJ, Cody R, Castaigne A, Perez A, Palensky J, Wittes J. The effect of spironolactone on morbidity and mortality in patients with severe heart failure. Randomized Aldactone Evaluation Study Investigators. N Engl J Med, 1999, 341: 709-717

19 Zannad F, McMurray JJ, Krum H, van Veldhuisen DJ, Swedberg K, Shi H, Vincent J, Pocock SJ, Pitt B, Group E-HS. Eplerenone in patients with systolic heart failure and mild symptoms. N Engl J Med, 2011, 364: 11-21

20 McCurley A, Jaffe IZ. Mineralocorticoid receptors in vascular function and disease. Mol Cell Endocrinol, 2012, 350: 256-265

21 Hillaert MA, Lentjes EG, Kemperman H, van der Graaf Y, Nathoe HM, Beygui F, Montalescot G, Doevendans PA, Wassink AM, van Belle E, Group SS. Aldosterone, atherosclerosis and vascular events in patients with stable coronary artery disease. Int J Cardiol, 2013, 167: 1929-1935

22 de Rita O, Hackam DG, Spence JD. Effects of aldosterone on human atherosclerosis: plasma aldosterone and progression of carotid plaque. Can J Cardiol, 2012, 28: 706-711

23 Milliez P, Girerd X, Plouin PF, Blacher J, Safar ME, Mourad JJ. Evidence for an increased rate of cardiovascular events in patients with primary aldosteronism. J Am Coll Cardiol, 2005, 45: 1243-1248

24 Weiss D, Taylor WR. Deoxycorticosterone acetate salt hypertension in apolipoprotein $\mathrm{E}^{-/-}$mice results in accelerated atherosclerosis: the role of angiotensin II. Hypertension, 2008, 51: 218-224

25 McGraw AP, Bagley J, Chen WS, Galayda C, Nickerson H, Armani A, Caprio M, Carmeliet P, Jaffe IZ. Aldosterone increases early atherosclerosis and promotes plaque inflammation through a placental growth factor-dependent mechanism. J Am Heart Assoc, 2013, 2: e000018

26 Briet M, Schiffrin EL. Vascular Actions of Aldosterone. J Vasc Res, 2012, 50: 89-99

27 Ward MR, Kanellakis P, Ramsey D, Funder J, Bobik A. Eplerenone suppresses constrictive remodeling and collagen accumulation after angioplasty in porcine coronary arteries. Circulation, 2001, 104: 467-472

28 Whaley-Connell A, Johnson MS, Sowers JR. Aldosterone: role in the cardiometabolic syndrome and resistant hypertension. Prog Cardiovasc Dis, 2010, 52: 401-409

29 Brown NJ. Aldosterone and vascular inflammation. Hypertension, 2008, 51: 161-167

30 Wakabayashi K, Suzuki H, Sato T, Iso Y, Katagiri T, Takeyama Y. Eplerenone suppresses neointimal formation after coronary stent implantation in swine. Int J Cardiol, 2006, 107: 260-266

31 Virdis A, Neves MF, Amiri F, Viel E, Touyz RM, Schiffrin EL. Spironolactone improves angiotensin-induced vascular changes and oxidative stress. Hypertension, 2002, 40: 504-510

32 Endemann DH, Touyz RM, Iglarz M, Savoia C, Schiffrin EL. Eplerenone prevents salt-induced vascular remodeling and cardiac fibrosis in stroke-prone spontaneously hypertensive rats. Hypertension, 2004, 43: 1252-1257

33 Sakurabayashi-Kitade S, Aoka Y, Nagashima H, Kasanuki H, Hagiwara N, Kawana M. Aldosterone blockade by Spironolactone improves the hypertensive vascular hypertrophy and remodeling in angiotensin II overproducing transgenic mice. Atherosclerosis, 2009, 206: 54-60

34 Preston IR, Sagliani KD, Warburton RR, Hill NS, Fanburg BL, Jaffe IZ. Mineralocorticoid receptor antagonism attenuates experimental pulmonary hypertension. Am J Physiol Lung Cell Mol Physiol, 2013,
304): L678-688

35 Keidar S, Hayek T, Kaplan M, Pavlotzky E, Hamoud S, Coleman R, Aviram M. Effect of eplerenone, a selective aldosterone blocker, on blood pressure, serum and macrophage oxidative stress, and atherosclerosis in apolipoprotein E-deficient mice. J Cardiovasc Pharmacol, 2003, 41: 955-963

36 Rajagopalan S, Duquaine D, King S, Pitt B, Patel P. Mineralocorticoid receptor antagonism in experimental atherosclerosis. Circulation, 2002, 105: 2212-2216

37 Takai S, Jin D, Muramatsu M, Kirimura K, Sakonjo H, Miyazaki M. Eplerenone inhibits atherosclerosis in nonhuman primates. Hypertension, 2005, 46: 1135-1139

38 Labuzek K, Liber S, Buldak L, Krupej-Kedzierska J, Machnik G, Bobrzyk M, Okopien B. Eplerenone mimics features of the alternative activation in macrophages obtained from patients with heart failure and healthy volunteers. Eur J Pharmacol, 2014, 726C: 96-108

39 Labuzek K, Liber S, Buldak L, Machnik G, Liber J, Okopien B. Eplerenone promotes alternative activation in human monocytederived macrophages. Pharmacol Rep, 2013, 65: 226-234

40 McCurley A, Pires PW, Bender SB, Aronovitz M, Zhao MJ, Metzger D, Chambon P, Hill MA, Dorrance AM, Mendelsohn ME, Jaffe IZ. Direct regulation of blood pressure by smooth muscle cell mineralocorticoid receptors. Nat Med, 2012, 18: 1429-1433

41 Pruthi D, McCurley A, Aronovitz M, Galayda C, Karumanchi SA, Jaffe IZ. Aldosterone promotes vascular remodeling by direct effects on smooth muscle cell mineralocorticoid receptors. Arterioscler Thromb Vasc Biol, 2014, 34: 355-364

42 Rautureau Y, Paradis P, Schiffrin EL. Cross-talk between aldosterone and angiotensin signaling in vascular smooth muscle cells. Steroids, 2011, 76: 834-839

43 Grossmann C, Gekle M. Interaction between mineralocorticoid receptor and epidermal growth factor receptor signaling. Mol Cell Endocrinol, 2012, 350: 235-241

44 Nguyen Dinh Cat A, Griol-Charhbili V, Loufrani L, Labat C, Benjamin L, Farman N, Lacolley P, Henrion D, Jaisser F. The endothelial mineralocorticoid receptor regulates vasoconstrictor tone and blood pressure. FASEB J, 2010, 24: 2454-2463

45 Schafer N, Lohmann C, Winnik S, van Tits LJ, Miranda MX, Vergopoulos A, Ruschitzka F, Nussberger J, Berger S, Luscher TF, Verrey F, Matter CM. Endothelial mineralocorticoid receptor activation mediates endothelial dysfunction in diet-induced obesity. Eur Heart J, 2013, 34: 3515-3524

46 Rickard AJ, Morgan J, Chrissobolis S, Miller AA, Sobey CG, Young MJ. Endothelial cell mineralocorticoid receptors regulate deoxycorticosterone/salt-mediated cardiac remodeling and vascular reactivity but not blood pressure. Hypertension, 2014, 63: 1033-1040

47 Caprio M, Newfell BG, la Sala A, Baur W, Fabbri A, Rosano G, Mendelsohn ME, Jaffe IZ. Functional mineralocorticoid receptors in human vascular endothelial cells regulate intercellular adhesion molecule-1 expression and promote leukocyte adhesion. Circ Res, 2008, 102: 1359-1367

48 Deuchar GA, McLean D, Hadoke PW, Brownstein DG, Webb DJ, Mullins JJ, Chapman K, Seckl JR, Kotelevtsev YV. 11betahydroxysteroid dehydrogenase type 2 deficiency accelerates atherogenesis and causes proinflammatory changes in the endothelium in Apo $E^{-1-}$ mice. Endocrinology, 2011, 152: 236-246

49 Iwashima F, Yoshimoto T, Minami I, Sakurada M, Hirono Y, Hirata Y. Aldosterone induces superoxide generation via Rac1 activation in endothelial cells. Endocrinology, 2008, 149: 1009-1014

50 Leopold JA, Dam A, Maron BA, Scribner AW, Liao R, Handy DE, Stanton RC, Pitt B, Loscalzo J. Aldosterone impairs vascular reactivity by decreasing glucose-6-phosphate dehydrogenase activity. Nat Med, 2007, 13: 189-197

51 Liu SL, Schmuck S, Chorazcyzewski JZ, Gros R, Feldman RD. Aldosterone regulates vascular reactivity: short-term effects mediated by phosphatidylinositol 3-kinase-dependent nitric oxide synthase activation. Circulation, 2003, 108: 2400-2406 
52 Nagata D, Takahashi M, Sawai K, Tagami T, Usui T, Shimatsu A, Hirata Y, Naruse M. Molecular mechanism of the inhibitory effect of aldosterone on endothelial NO synthase activity. Hypertension, 2006, 48: $165-171$

53 Bene NC, Alcaide P, Wortis HH, Jaffe IZ. Mineralocorticoid receptors in immune cells: emerging role in cardiovascular disease. Steroids, 2014, doi: 10.1016/j.steroids.2014.04.005

54 Herrada AA, Contreras FJ, Marini NP, Amador CA, Gonzalez PA,
Cortes CM, Riedel CA, Carvajal CA, Figueroa F, Michea LF, Fardella CE, Kalergis AM. Aldosterone promotes autoimmune damage by enhancing Th17-mediated immunity. J Immunol, 2010, 184: 191-202

55 Besedovsky L, Linz B, Born J, Lange T. Mineralocorticoid receptor signaling reduces numbers of circulating human naive $\mathrm{T}$ cells and increases their CD62L, CCR7, and CXCR4 expression. Eur J Immunol, 2014, 44: 1759-1769

Open Access This article is distributed under the terms of the Creative Commons Attribution License which permits any use, distribution, and reproduction in any medium, provided the original author(s) and source are credited. 